\title{
RECENT GRAFT MATERIALS IN EXPERIMENTAL STUDY STAGE USED IN THE TREATMENT OF NASAL SEPTUM PERFORATION: A REVIEW
}

\author{
Serdal Celik, Yasemin Gunduz, M. Tayyar Kalcioglu \\ Department of Otorhinolaryngology, Faculty of Medicine, Istanbul Medeniyet University, \\ Istanbul, Turkey
}

\begin{abstract}
Various conservative approaches are being used for the treatment of nasal septum perforations (NSPs); however, for permanent results, it is essential to use surgical methods. For this purpose, many various surgical methods have been tried, but a satisfactory method has not yet been determined. In order to eliminate the commonly encountered failure of surgical methods, new graft materials combined with flaps have been tried recently and successful results have been reported.

In this review, we examined the scientific literature using Medline, PubMed, and Google by using the keywords "nasal septum perforation", "animal study", and "graft". According to the results obtained, we compiled graft materials that have been used in experimental stage related to this subject and reported to have achieved successful results, especially when combined with flaps. These materials seem promising for the successful closure of the hard-to-treat NSPs.
\end{abstract}

Keywords: nasal septum perforation, treatment, graft materials

Address for correspondence:

M. Tayyar Kalcioglu

Department of Otorhinolaryngology, Faculty of Medicine

Istanbul Medeniyet University

Dumlupinar D100 Karayolu No:98

34720 Kadıköy

Istanbul

Turkey

e-mail: mtkalcioglu@hotmail.com

Received: June 8, 2021

Accepted: June 22, 2021

\section{INTRODUCTION}

Nasal septum perforation (NSP) is the composite tissue loss including the mucous membrane and the bone or cartilage participating in the structure of the nasal septum (1). The most common cause in etiology is septal surgery performed earlier. Other reasons may be: traumatic causes such as septal hematoma, nose picking habit, nasal cauterization performed after nosebleeds, and nasotracheal intubation; nasal spray use, cocaine use; inflammatory dis- 
eases such as vasculitis, sarcoidosis, and Wegener granulomatosis; and infections such as tuberculosis, leprosy, syphilis, and diphtheria $(2,3)$.

Septal button prosthesis has been used in the non-surgical treatment of NSP, but long-term results included problems such as displacement of the prosthesis and foreign body reactions (4). Today, instead of extensive surgical methods, conservative methods and use of prostheses are options that can be applied especially in patients with active granulomatous disease and those who do not accept surgery. However, surgical methods are required for the conclusive result. So far, although many surgical methods have been tried for this purpose, they have not been successful enough.

In order to increase surgical success, animal models have been created in recent years and new graft materials combined with flaps already used in practice have been tried. The results are promising for the successful treatment of NSP.

\section{CURRENT RESEARCH GOALS AND RESULTS}

We examined the scientific literature using Medline, PubMed, and Google with the keywords "nasal septum perforation", "animal study", and "graft". Our deadline for the search was September 2020.

To date, many grafts that have been used in the treatment have been identified in the literature, most of which are autologous grafts used on humans. The success of grafts used in NSP is basically linked to generation of a vector force for mucosal progression, which is necessary for the onset of recovery. The graft variety is as wide as the flap variety. The grafts described in the literature to date include mastoid periosteum, temporalis fascia graft, septal bone and cartilage, inferior concha, auricular conchal cartilage, free radial forearm fascial flap, acellular human dermal allograft, bovine perichondrium, titanium membrane, and even pig small intestine mucosa (5-10).

In addition to the grafts used in humans, promising graft materials that are still in experimental stage are also being reported. In the literature, there are 5 studies in which new materials are tried by cre- ating nasal septum perforations in experimental animals.

Yücebaş et al. conducted an experimental study in which they used polypropylene mesh for this purpose (11). The rabbits were divided into two groups. In one group they repaired NSP using polypropylene mesh in addition to interpositional mucosal flap, while in the other group they used only mucosal flap; and as a result, they reported that the perforation in the polypropylene mesh group was completely closed, and that lower degrees of significant fibrosis, inflammatory response, infection, and microabscesses were observed as well. As a result, they reported that this material had good tissue compatibility and could be safely used as a graft for perforation repair.

Martinez et al. investigated the effect of bacterial cellulose on NSP in rabbits (12). They reported that they applied fibrin glue as the bacterial cellulose tissue adhesive to the NSP region they created in rabbits, and as a result, the perforation remained in the rabbits in the control group whereas the perforation was closed in about half of the rabbits in the experimental group. However the difference was not statistically significant, which may be attributed to the small number of rabbits in the study groups.

Yıldırım et al. investigated the NSP repair model with high-density porous polyethylene (HDPP) covered with fascia lata (13). In this study, HDPP covered with fascia lata was placed in the experimental group and only HDPP was placed in the control group. They histologically and clinically evaluated perforation, resorption of the material used, and graft status, and reported that perforation was closed in $90 \%$ of those in the group with HDPP covered with fascia lata, while success remained at $40 \%$ in the group using only HDPP. In this study, fascia lata was obtained with an additional surgical procedure. This increases the cost. Besides, the additional surgical intervention required for fascia lata to be taken will increase the likelihood of complications.

In their study, Mola et al. created defects in nasal septa and used acellular dermal matrix (Alloderm), Dacron, Gore-Tex, and autologous cartilage grafts in these defects to evaluate the reactions of these substances (14). In their study in which they placed these materials after removing $3 \times 10 \mathrm{~mm}$ of septal cartilage 
without damaging the mucous membranes of rabbits whose nasal septal cartilages were exposed, they reported that acute inflammation reactions mostly occurred with Gore-Tex, and that there was no difference among the groups with respect to chronic inflammation. Given the reaction rate and tissue compatibility, they reported that cartilage performed the best, followed by Alloderm. Even though it developed acute inflammation, they reported that Dacron had success in terms of tissue compatibility.

In a study, Çelik investigated the efficacy of hyaluronic acid (HA) and platelet-rich fibrin (PRF) on nasal septum perforation of rabbits (15). A nasal septum perforation model was created in a total of 24 rabbits divided into 4 groups, each of which included 6 rabbits. No additional intervention was done in the first group that was the control group. In the 2nd group, septum perforation was closed by using $\mathrm{MeroGel}^{\circledR}$, which contained pure HA. In the 3rd group, the blood drawn from the rabbits was centrifuged and the perforations were closed with PRF. In the 4th group, perforations were closed using both HA and PRF together. According to the results obtained, none of the septum perforations in Group 1 showed complete closure. In the 2 nd group in which HA was used and in the 3rd Group in which PRF was used, 3 rabbits (50\%) from each group had a complete closure of their nasal septum perforations. A closure of more than $50 \%$ was detected in septum perforations of other rabbits. In the last group, which is the 4th group, 4 rabbits (66.6\%) had complete closure of their nasal septum perforations, while other rabbits had a closure of more than $50 \%$ in their nasal septum perforations. These findings of complete perforation closure could not be statistically supported. This was attributed to the small number of rabbits used. When perforations were evaluated in terms of closure of more than 50\%, statistically significant results were reported. When groups containing HA in the treatment (groups 2 and 4 ) were evaluated histopathologically, it was reported that foreign body reaction and fibrosis were seen in both groups, but there was no statistically significant difference in postoperative secretion and inflammation compared to those of the control group. These findings suggest that, although the material used has a risk of creating a foreign body reaction, its tissue compatibility is high. In the group where PRF, which can be classi- fied as an autograft, was used, observing no foreign body reactions in any of the specimens was not an unexpected result.

\section{CONCLUSION}

NSP can be a disease whose treatment is especially avoided by physicians because the consequences can be unsatisfactory for the patient. For this reason, the idea of the need for new materials that can be used in this regard, which are easy to obtain and have high tissue compatibility, arises. As it can be seen, many new grafts have been used experimentally for the reconstruction of NSP, and the search for new materials that may have a higher success in this regard continues. Novel animal studies or clinical trials on this subject are needed.

\section{REFERENCES}

1. Re M, Paolucci L, Romeo R, Mallardi V. Surgical treatment of nasal septal perforations. Our experience. Acta Otorhinolaryngol Ital. 2006; 26(2):102-9.

2. Metzinger SE, Guerra AB. Diagnosing and treating nasal septal perforations. Aesthetic Surg J. 2005; 25(5):524-9. doi: 10.1016/j.asj.2005.06.002.

3. Vignes $\mathrm{S}$, Chaillet $\mathrm{M}, \mathrm{Cabane}$ J, Piette JC. Nasal septal perforation and systemic disease. Rev Med Inteme. 2002; 23(11):919-26. doi: 10.1016/ s0248-8663(02)00711-7.

4. Goodman WS, Strelzow W. The surgical closure of nasoseptal perforations. Laryngoscope. 1982;92(2):121-4. doi: 10.1002/lary.1982.92.2.121.

5. Tastan E, Aydogan F, Aydin E, Can IH, Demirci M, Uzunkulaoglu $\mathrm{H}$, et al. Inferior turbinate composite graft for repair of nasal septal perforation. Am J Rhinol Allergy. 2012;26(3):237-42. doi: 10.2500/ ajra.2012.26.3765.

6. Ambro BT, Zimmerman J, Rosenthal M, Pribitkin EA. Nasal septal perforation repair with porcine small intestinal submucosa. Arch Facial Plast Surg. 2003;5(6):528-9. doi: 10.1001/archfaci.5.6.528.

7. Fairbanks DN, Fairbanks GR. Nasal septal perforation: prevention and management. Ann Plast Surg. 1980;5(6):452-9. doi: 10.1097/00000637-198012000-00007.

8. Nunez-Fernandez D, Vokurka J, Chrobok V. Bone and temporal fascia graft for the closure of septal perforation. J Laryngol Otol. 1998; 112(12):1167-71. doi: $10.1017 / \mathrm{s} 0022215100142744$. 
9. Teichgraeber JF, Russo RC. The management of septal perforations. Plast Reconstr Surg. 1993;91(2):229-35. doi: 10.1097/00006534-199302000-00004.

10. Kridel RWH, Appling WD, Wright WK. Septal perforation closure utilising the external septorhinoplasty approach. Arch Otolaryngol Head Neck Surg. 1986;112(2):168-72. doi: 10.1001/ archotol.1986.03780020048011.

11. Yücebaş K, Taşkın Ü, Oktay MF, Tansuker HD, Erdil M, Altınay S, et al. Polypropylene mesh for nasal septal perforation repair: an experimental study. Eur Arch Otorhinolaryngol. 2017;274(1):2616. doi: 10.1007/s00405-016-4237-5.

12. Martinez Neto EE, Lutaif Dolci JE. Nasal septal perforation closure with bacterial cellulose in rabbits. Braz J Otorhinolaryngol. 2010; 76(4):442-9.
13. Yildırım G, Onar V, Sayın I, Onol SD, Aydin T. The reconstruction of nasal septal perforation with density porous polyethlene covered with fascia lata: an experimental study on rabbit model. Clin Exp Otorhinolaryngol. 2011; 4(3):137-41. doi: 10.3342/ ceo.2011.4.3.137.

14. Mola F, Keskin G, Ozturk M, Muezzinoglu B. The comparison of acellular dermal matric (Alloderm), Dacron, Gore-Tex, and autologous cartilage graft materials in an experimental animal model for nasal septal repair surgery. Am J Rhinol. 2007;21(3):330-334. doi:10.2500/ajr.2007.21.2975.

15. Celik S. The effect of platelet-rich fibrin and hyaluronic acid on perforation of nasal septum in rabbits: An experimental study. Master thesis. Istanbul Medeniyet University Faculty of Medicine, Istanbul; 2020. 